\title{
INEQUALITIES VIA LAGRANGE MULTIPLIERS
}

\author{
W. T. SULAIMAN \\ Zarka Private University \\ P O. Box 150863 \\ Zarka 13115, JORDAN
}

(Received August 8, 1993 and in revised form June 6, 1996)

ABSTRACT. An easy method is obtained to prove many inequalities using Lagrange mutipliers.

KEY WORDS AND PHRASES: Inequalities.

1991 AMS SUBJECT CLASSIFICATION CODES: 90C50, $980 \mathrm{C} 39$.

\section{INTRODUCTION}

Let us assume that $d_{1}, \ldots, d_{n}$ are unit perpendicular vectors in an $n$-dimensional space $X$. In particular $d_{1}, d_{2}$, and $d_{3}$ are the unit perpendicular vectors $i, j$, and $k$ in the 3-dimensional space. Any vector $v$ in $X$ is usually uniquely written in the form

$$
v=\sum_{i=1}^{n} \lambda_{i} d_{i}
$$

for scalars $\lambda_{2}$. We define

$$
\nabla f\left(x_{1}, \ldots, x_{n}\right)=\sum_{i=1}^{n} f_{x_{i}}\left(x_{1}, \ldots, x_{n}\right) d_{i}, \quad f_{x}=\frac{\partial}{\partial x} .
$$

Kapur and Kumar (1986), have used the principle of dynamic programming to prove major inequalities due to Shannon, Renyi, and Holder, see [1]. In this note we give a new method using Lagrange multipliers.

\section{SHANNON'S INEQUALITY}

THEOREM 2.1. Given $\sum_{i=1}^{n} p_{i}=a, \sum_{i=1}^{n} q_{i}=b$, then

$$
a \ln (a / b) \leq \sum_{i=1}^{n} p_{i} \ln \left(p_{i} / q_{i}\right), \quad p_{2}, q_{2} \geq 0 .
$$

The equality holds iff $p_{\imath}=q_{2}$ for each $i$.

PROOF. Let the $q_{2}$ 's and $a$ be fixed; set

$$
f\left(p_{1}, \ldots, p_{n}\right)=\sum_{i=1}^{n} p_{\imath} \ln \left(p_{\imath} / q_{2}\right) ; \quad p_{\imath}, q_{\imath} \geq 0,
$$

we aim to minimize $f$ subject to the constraint

$$
g\left(p_{1}, \ldots, p_{n}\right)=\sum_{i=1}^{n} p_{\imath}-a=0 .
$$


There is a minimum achieved where $\nabla f=\lambda \nabla g$ because $g$ is linear and $f$ is convex, since its second order partials are all non-negative

$$
\begin{aligned}
\nabla f=\lambda \nabla g & \Rightarrow \sum_{i=1}^{n}\left\{1+\ln \left(p_{\imath} / q_{\imath}\right)\right\} d_{\imath}=\lambda \sum_{i=1}^{n} d_{i} \\
& \Rightarrow 1+\ln \left(p_{i} / q_{2}\right)=\lambda \\
& \Rightarrow \frac{p_{1}}{q_{1}}=\frac{p_{2}}{q_{2}}=\cdots=\frac{p_{n}}{q_{n}}=\frac{\sum a_{\imath}}{\sum b_{\imath}}=\frac{a}{b} .
\end{aligned}
$$

Therefore

$$
\min \sum_{\imath=1}^{n} p_{\imath} \ln \left(p_{\imath} / q_{\imath}\right)=\ln (a / b) \sum_{i=1}^{n} p_{\imath}=a \ln (a / b)
$$

or

$$
a \ln (a / b) \leq \sum_{i=1}^{n} p_{i} \ln \left(p_{2} / q_{\imath}\right)
$$

If $a=b=1$, we get Shannon's inequality

$$
\sum_{i=1}^{n} p_{i} \ln \left(p_{\imath} / q_{i}\right) \geq 0 \text { and } \sum_{i=1}^{n} p_{\imath} \ln \left(p_{i} / q_{i}\right)=0 \text { iff } p_{\imath}=q_{i} \text { for each } i \text {. }
$$

\section{RENYI'S INEQUALITY}

THEOREM 3.1. Given $\sum_{i=1}^{n} a_{\imath}=a, \sum_{i=1}^{n} b_{\imath}=b$, then

$$
\frac{1}{\alpha-1}\left(a^{\alpha} b^{1-\alpha}-a\right) \leq \sum_{i=1}^{n} \frac{1}{\alpha-1}\left(p_{2}^{\alpha} q_{2}^{1-\alpha}-p_{i}\right), \quad p_{i}, q_{2} \geq 0,0<\alpha \neq 1 .
$$

The equality holds iff $p_{2}=q_{i}$ for each $i$.

PROOF. Let the $q_{i}$ 's and $a$ be fixed and write

$$
\begin{gathered}
f\left(p_{1}, \ldots, p_{n}\right)=\sum_{i=1}^{n} \frac{1}{\alpha-1} p_{\imath}^{\alpha} q_{\imath}^{1-\alpha}, \quad g\left(p_{1}, \ldots, p_{n}\right)=\sum_{i=1}^{n} p_{i}-\alpha=0 \\
\nabla f=\lambda \nabla g
\end{gathered}
$$

by the convexity of $f$ and linearity of $g$. Hence

$$
\frac{1}{\alpha-1} a^{\alpha} b^{1-\alpha} \leq \sum_{\imath=1}^{n} \frac{1}{\alpha-1} p_{\imath}^{\alpha} q_{\imath}^{1-\alpha}
$$

If $a=b=1$, we get Renyi's inequality

$$
\frac{1}{\alpha-1}\left(\sum_{\imath=1}^{n} p_{\imath}^{\alpha} q_{\imath}^{1-\alpha}-1\right) \geq 0
$$

\section{HOLDER'S INEQUALITY}

THEOREM 4.1. Given $\sum_{i=1}^{n} a_{i}^{p}=A, \sum_{i=1}^{n} b_{i}^{q}=B, \sum_{i=1}^{n} a_{i} b_{i}=C, a_{i}, b_{\imath} \geq 0, p, q>1, \frac{1}{p}+\frac{1}{q}=1$, 
then

$$
C \leq A^{1 / p} B^{1 / q} .
$$

PROOF. This follows from Renyi's inequality, taking $\alpha=1 / p, a_{i}=p_{i}^{p}, b_{i}=q_{i}^{q}$, or, we prove the result directly as follows:

let the $a_{\imath}$ 's and $C$ be fixed and write

$$
\begin{gathered}
f\left(b_{1}, \ldots, b_{n}\right)=A^{q / p} \sum_{i=1}^{n} b_{\imath}^{q}, g\left(b_{1}, \ldots, b_{n}\right)=\sum_{i=1}^{n} a_{\imath} b_{\imath}-C=0 \\
\nabla f=\lambda \nabla g \Rightarrow q A^{q / p} \sum_{i=1}^{n} b_{\imath}^{q-1} d_{\imath}=\lambda \sum_{\imath=1}^{n} a_{\imath} d_{\imath} \\
\Rightarrow A^{q / p} b_{\imath}^{q-1}=(\lambda / q) a_{\imath} \\
(4.2) \Rightarrow A^{q / p}=(\lambda / q) C,
\end{gathered}
$$

and

$$
A^{q} B=(\lambda / q) A, \quad \text { as } \quad p(q-1)=q
$$

$$
\text { (4.3) \& (4.4) } \Rightarrow \lambda / q=C^{q-1} \text {. }
$$

Therefore, by the convexity of $f$ and linearity of $g$,

$$
\min \left(A^{q / p} B\right)=C^{q},
$$

or

$$
C \leq A^{1 / p} B^{1 / q}
$$

\section{GENERALIZATIONS OF HOLDER'S INEQUALITY}

THEOREM 5.1. Given $\sum_{i=1}^{n} a_{i}^{p}=A, \sum_{i=1}^{n} b_{i}^{q}=B, \sum_{i=1}^{n} c_{i}^{r}=C$, and $\sum_{i=1}^{n} a_{i} b_{i} c_{\imath}=D, a_{\imath}, b_{i}, c_{i} \geq 0$, $\frac{1}{p}+\frac{1}{q}+\frac{1}{r}=1$, then

$$
D \leq a^{1 / p} B^{1 / q} C^{1 / \tau} .
$$

PROOF. This follows by an easy application of Holder's inequality:

$$
\begin{aligned}
\sum_{i=1}^{n} a_{i} b_{i} c_{i} & \leq\left[\sum_{i=1}^{n}\left(a_{i} b_{i}\right)^{\frac{r}{r-1}}\right]^{1-\frac{1}{r}} C^{\frac{1}{r}} \\
& =\left[\sum_{i=1}^{n}\left(a_{i} b_{i}\right)^{\frac{p q}{p+q}}\right]^{\frac{1}{p}+\frac{1}{q}} C^{\frac{1}{r}} \\
& \leq\left[\sum_{i=1}^{n}\left(a_{i}^{\frac{p q}{p+q}}\right)^{\frac{p+q}{q}}\right]^{\frac{p}{p+q}\left(\frac{p+q}{p q}\right)}\left[\sum_{i=1}^{n}\left(b_{i}^{\frac{p q}{p+q}}\right)^{\frac{p+q}{p}}\right]^{\frac{p}{p+q}\left(\frac{p+q}{p q}\right)} C^{\frac{1}{r}} \\
& =A^{\frac{1}{p}} B^{\frac{1}{q}} C^{\frac{1}{r}} .
\end{aligned}
$$

\section{MINKOWSKI'S INEQUALITY}

THEOREM 6.1. Given $\sum_{i=1}^{n} a_{i}^{p}=A, \sum_{i=1}^{n} b_{i}^{p}=B$, and $\sum_{i=1}^{n}\left(a_{i}+a_{i}\right)^{p}=C, q_{i}, b_{i} \geq 0, p \geq 1$, then

$$
C^{\frac{1}{p}} \leq A^{\frac{1}{p}}+B^{\frac{1}{p}} .
$$

PROOF. Let the $b_{\imath}$ 's and $A$ be fixed and write 
Therefore,

$$
\begin{gathered}
f\left(a_{1}, \ldots, a_{n}\right)=\sum_{i=1}^{n}\left(a_{\imath}+b_{\imath}\right)^{p}, \quad g\left(a_{1}, \ldots, a_{n}\right)=\sum_{i=1}^{n} a_{\imath}^{p}-A=0 \\
\nabla f=\mu \nabla g \\
\Rightarrow \sum_{\imath=1}^{n} p\left(a_{i}+b_{i}\right)^{p-1} d_{\imath}=\mu \sum_{\imath=1}^{n} p a_{\imath}^{p-1} d_{\imath} \\
\Rightarrow\left(a_{\imath}+b_{\imath}\right)^{p-1}=\mu a_{\imath}^{p-1} \\
\Rightarrow \frac{b_{1}}{a_{1}}=\cdots=\frac{b_{n}}{a_{n}}=C .
\end{gathered}
$$

$$
\begin{aligned}
\max C^{\frac{1}{p}} & =\left[\sum_{i=1}^{n}\left(a_{\imath}+c a_{\imath}\right)^{p}\right]^{\frac{1}{p}} \\
& =(1+c) A^{\frac{1}{p}} \\
& =A^{\frac{1}{p}}+c A^{\frac{1}{p}} \\
& =A^{\frac{1}{p}}+B^{\frac{1}{p}}
\end{aligned}
$$

or

$$
C^{\frac{1}{p}} \leq A^{\frac{1}{p}}+B^{\frac{1}{p}}
$$

\section{ARITHMETIC-GEOMETRIC-MEAN INEQUALITY}

THEOREM 7.1.

$$
\left(\prod_{i=1}^{n} x_{i}\right)^{\frac{1}{n}} \leq \frac{1}{n} \sum_{i=1}^{n} x_{i}
$$

PROOF. Write

$$
f\left(x_{1}, \ldots, x_{n}\right)=x_{1} x_{2} \ldots x_{n}=y, \quad g\left(x_{1}, \ldots, x_{n}\right)=\frac{1}{n} \sum_{i=1}^{n} x_{i}-C=0 .
$$

Let $C$ be fixed, we have

$$
\begin{aligned}
\nabla f=\mu \nabla g & \Rightarrow \sum_{i=1}^{n} \frac{y}{x_{i}} d_{i}=\frac{\mu}{n} \sum_{i=1}^{n} d_{i} \\
& \Rightarrow x_{i}=\frac{n}{\mu} y \\
& \Rightarrow C=\frac{n}{\mu} y .
\end{aligned}
$$

Therefore

$$
\max y^{\frac{1}{n}}=\frac{n}{\mu} y=C
$$

or

$$
\left(\prod_{\imath=1}^{n} x_{\imath}\right)^{\frac{1}{n}} \leq \frac{1}{n} \sum_{\imath=1}^{n} x_{\imath}
$$

ACKNOWLEDGMENT. The author is so grateful to the referee for his kind remarks, suggestions, and improvements of this paper.

\section{REFERENCES}

[1] KAPUR, J.N., KUMAR, V. and KUMAR, U., A measure of mutual divergence among a number of probability distributions, Internat. J. Math. \& Math. Sci., 10 (1987), 597-608. 


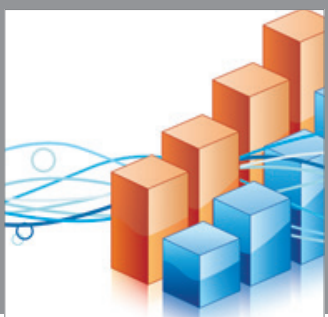

Advances in

Operations Research

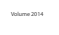

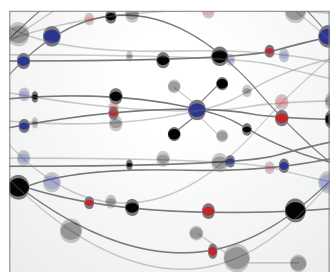

\section{The Scientific} World Journal
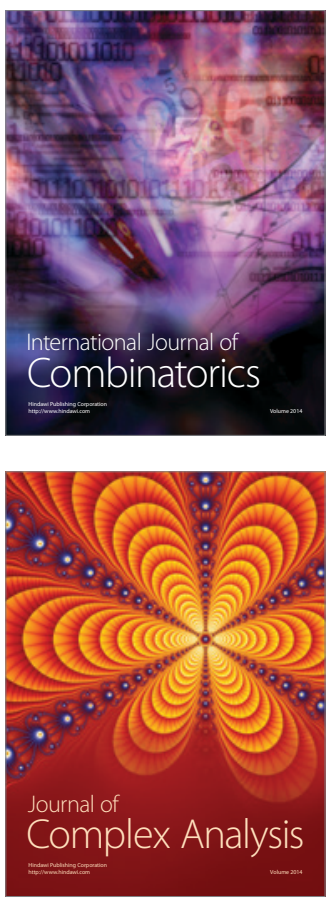

International Journal of

Mathematics and

Mathematical

Sciences
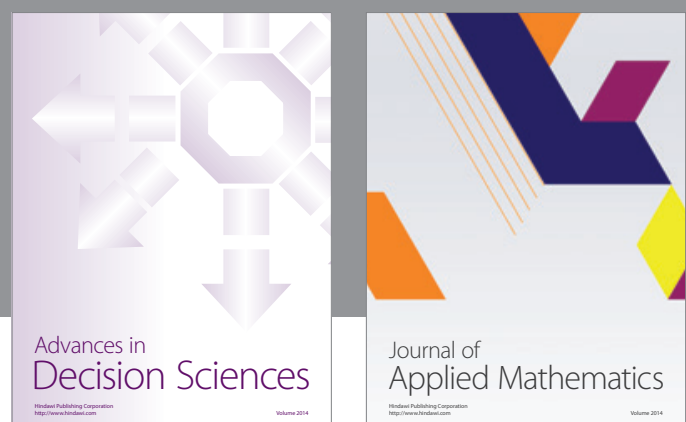

Journal of

Applied Mathematics
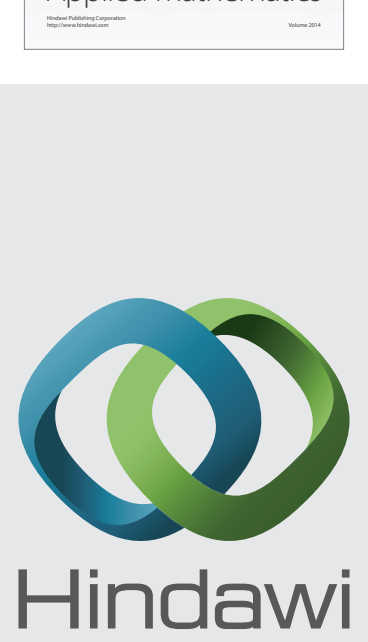

Submit your manuscripts at http://www.hindawi.com
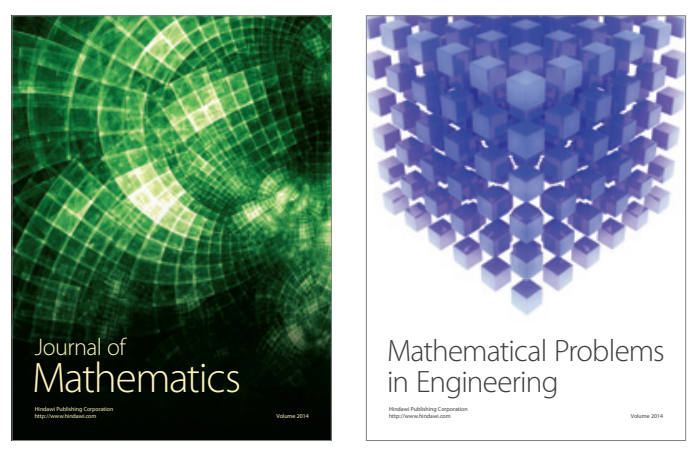

Mathematical Problems in Engineering
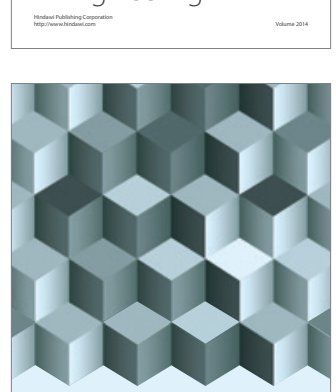

Journal of

Function Spaces
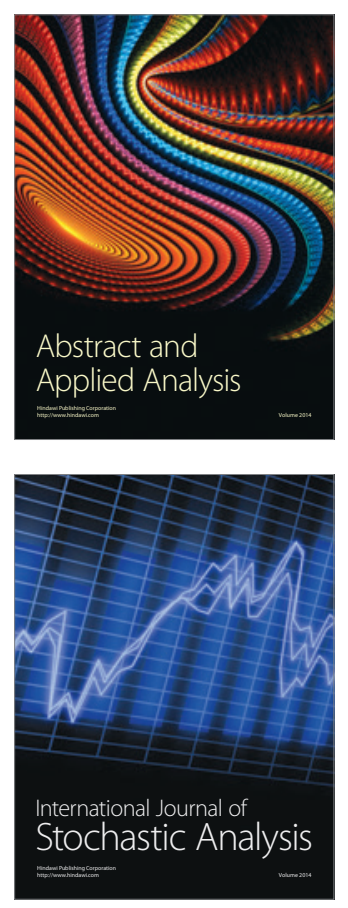

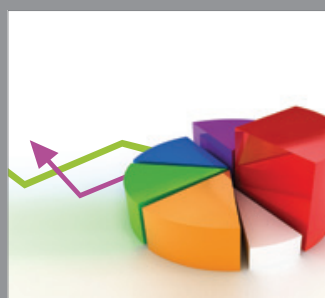

ournal of

Probability and Statistics

Promensencen
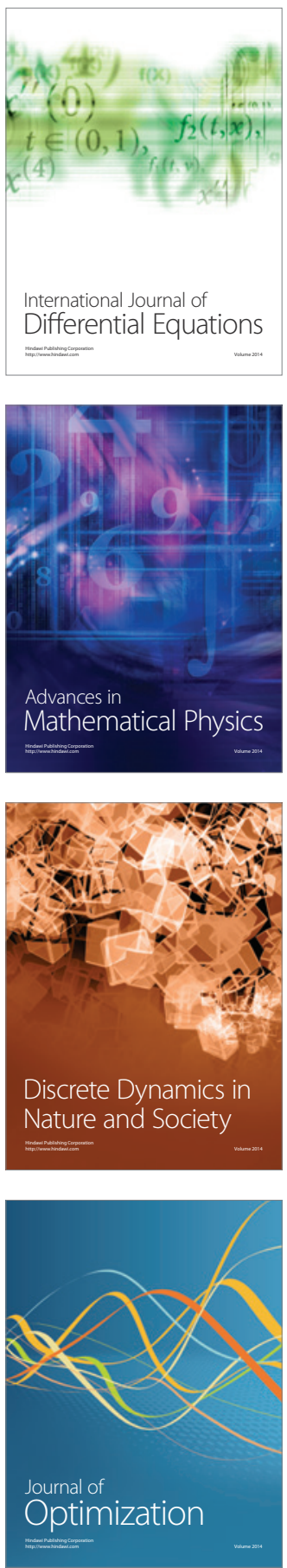